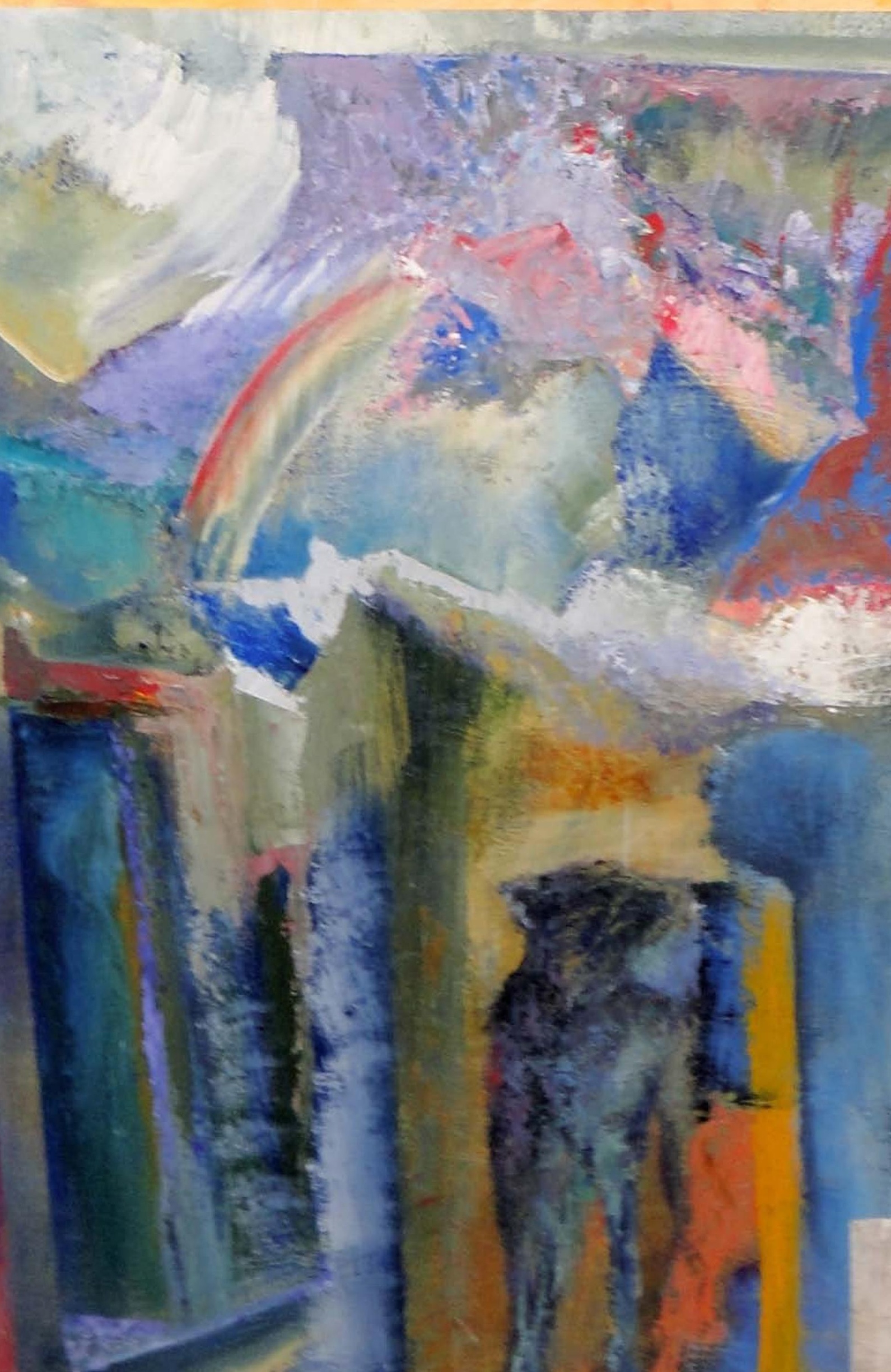




\title{
7 Efecto psicosocial de la migración en los alumnos del instituto Ángel G. Hernández y su incidencia en el rendimiento educativo
}

Lesl María Cardozo*

José AdÁn GonZÁles**

\begin{abstract}
RESUMEN. La migración es uno de los fenómenos demográficos que afecta la integración de las familias hondureñas. Aramecina, Valle es un pueblo pequeño cerca de la frontera con El Salvador, en donde uno de los mayores problemas es la falta de fuentes de trabajo situación que ha generado una fuerte migración de gran parte de la población como alternativa de solución a sus problemas económicos. El impacto de la emigración, afecta a los jóvenes sobre todo cuando uno o ambos padres emigran, y quedan a cargo de uno de ellos, por lo general de la mamá, o en su defecto un familiar cercano, quien asume el papel de madre y padre en su educación, y además de la formación del carácter. A través de este estudio se puede apreciar como el fenómeno de la migración influye en el rendimiento académico de los y las estudian-tes, aunque después de pasar un largo tiempo algunos logran nivelarse académicamente, también se puede apreciar que su ausencia incide considerablemente en su salud tanto física como mental afectando su conducta presentando niveles de agresividad, inseguridad y falta de afecto. Hoy en día la tecnología juega un papel muy importante ya que la comunicación con sus padres es más fácil permitiendo relaciones más estrechas, lo que les ayuda a sobrellevar su ausencia.
\end{abstract}

Palabras clave: migración, rendimiento educativo.

SUMMARY. Migration is a demographic phenomenon that affects the integration of Honduran families. Aramecina, Valle is a small town near the border with El Salvador, where one of the biggest problems is lack of jobs, a situation that has generated a strong migration of much of the population as an alternative for the solution to their economic problems. The impact of migration affects young people especially when one or both parents migrate, and they are left in charge of one of them, usually the mother, or failing that a close family member, who assumes the role of mother and father in their education and are also responsible for the character formation. Through this study it can be seen how the phenomenon of migration influences the academic performance of the students, although after spending a long time, some achieve academic standards, you can also see that their absence impacts considerably on both physical and mental health affecting their conduct and presenting levels of aggression, insecurity and lack of affection. Today, technology plays an important role as communication with parents is easier by allowing closer relationships, helping them cope with their absence.

Keywords: migration, educational performance.

\section{Introducción}

La migración es uno de los fenómenos demográficos que afecta la integración de las familias hondureñas. Migrar implica dejar hogar, lugares, afectos, tradiciones, todo con el afán de buscar mejores condiciones de vida, pero con frecuencia implica desintegración familiar que afecta la vida emocional y social de sus integrantes.
Una de las causas que motiva la migración entre los hondureños y hondureñas es la falta de fuentes de empleo y la crítica situación económica que se vive, misma que motiva que al menos un miembro de la familia se separe en busca de trabajo y de una vida mejor para implementar la confianza familiar entre padres e hijos.

A través de los tiempos el concepto de familia ha sido modificado desde el que correspondía a una estruc- 
turación de miembros férreamente unidos, amados, protegidos con la figura materna y paterna muy bien identificada y entregada a cuidar de sus hijos, hijas y hogar, por aquella en donde el padre o madre responsable hace sacrificios y se aleja del hogar por darle a su familia lo necesario para salir adelante, aunque esto signifique el privarles de su presencia y consejos por largos periodos de tiempo; en este sentido el factor económico ha sido determinante.

Aramecina es un pueblo pequeño en donde no existen fuentes de trabajo, ante las limitaciones económicas gran parte de su población ha recurrido a la migración como alternativa de solución a su problemática, generalmente migran hacia Estados Unidos en forma ilegal, (mojados), pero por la crisis económica global hoy también se han abierto los horizontes para viajar a trabajar a España, sólo que aquí lo están haciendo de forma legal. Esta situación ha generado modificaciones en la estructura de la población del municipio sobre todo porque la PEA es la que está migrando y en el municipio permanecen las personas de avanzada edad (abuelos y abuelas) y los menores de edad, niños, niñas y jóvenes, lo que ha hecho que Aramecina carezca de fuerza de trabajo.

En el contexto educativo el impacto de la migración está afectando seriamente a los y las jóvenes de la comunidad de Aramecina, departamento de Valle; cuando uno o ambos padres migran sobre todo por razones de orden económico, en tal sentido los hijos quedan a cargo de la mamá, o en su defecto de un familiar cercano, quien asume el papel de madre y padre en su educación, y además de la formación del carácter de los y las jóvenes, niños y niñas.

Esta investigación va encaminada a identificar los factores psicosociales que afectan el rendimiento educativo de las y los alumnos del Instituto Ángel G. Hernández, del municipio de Aramecina, departamento de Valle, cuyos padres han emigrado hacia las grandes ciudades del país o el extranjero, dejándolos solos por largos periodos de tiempo y algunas veces incluso hasta en el abandono.

\section{Metodología}

La investigación se realizó mediante un proceso participativo de consulta, con la revisión documental secundaria, relacionada con los registros académicos y disciplinarios de diferentes cursos y secciones de la comunidad educativa del Instituto "Ángel G. Hernández".

Se estructuraron instrumentos recolectores de información cuantitativa y cualitativa, la que se obtuvo a través de técnicas como encuestas a los estudiantes del instituto, entrevistas a informantes claves, grupos focales y entrevistas a profundidad a los miembros de las familias de los y las alumnas con padres migrantes; éstos instrumentos permitieron identificar las relaciones de apoyo para el educando.

El estudio se realizó teniendo como base los datos de los alumnos/as matriculados entre los años 2007 al año 2010.

Se utilizó como fuente de datos secundaria los documentos proporcionados por la secretaría del instituto para obtener información de los alumnos matriculados en el 2010, también de los registros de rendimiento académico de cada alumno(a), informes y registros del departamento de Orientación y Consejería del instituto, además datos demográficos proporcionados por el INE y se recolectaron datos primarios a través de entrevistas individuales y colectivas con los alumnos y grupos focales.

\section{Antecedentes generales}

\subsection{Ubicación geográfica del municipio de Aramecina}

El municipio de Aramecina está ubicado en la parte norte del departamento de Valle, cerca de la frontera con la república de El Salvador. Su territorio tiene una extensión de $108.8 \mathrm{Km}^{2}$, sus límites colindan al norte con el municipio de Caridad, al sur con Goascorán y Langue, al este con Curarén (del departamento de Francisco Morazán) y parte de Langue, al oeste con El Salvador (ver mapa No.1). 
Mapa No. 1. Aramecina: ubicación geográfica

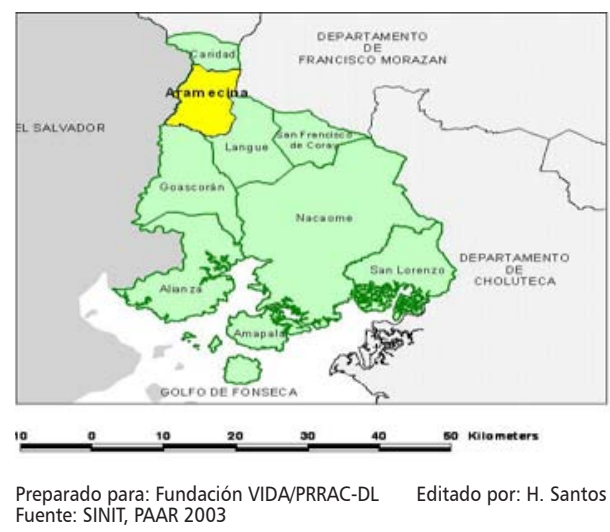

La densidad poblacional al 2010 era de 59 habitantes/ $\mathrm{km} 2$, con $17 \%$ de la población del municipio residiendo en la cabecera municipal (INE 2010).

El municipio de Aramecina, está conformado por 11 aldeas y la cabecera municipal: El Cantil, El Pedregal, El Tablón, La Peña, Las Pozas, Los Terrenos, Macuelizo, Sampito, Santa Lucía, Solubre y Tierra Blanca y la cabecera municipal, además de 60 caseríos, el resto de la población vive en caseríos con un promedio de 80 habitantes.

\subsection{Breve reseña histórica del municipio de Aramecina}

Aramecina se fundó en 1578, en la época de la colonia y obtuvo el título de municipio, concedido por la Capitanía General de Guatemala y renovado el 12 de febrero de 1858 en Comayagua donde a raíz del descubrimiento de ricos minerales en el Cerro Apasapo, tomó su primer nombre.

En 1969, este municipio fue invadido por el ejército salvadoreño en lo que se conoce como la Guerra del 69 lo que tuvo grandes repercusiones en el desarrollo del municipio ya que sus caseríos fueron saqueados, obligando a la mayor parte de la población a emigrar a otros lugares del país, quedando desierta la comunidad trayendo consigo el atraso al desarrollo local.

La cabecera municipal lleva el mismo nombre del municipio, Aramecina, el cual significa en lengua mejicana: "Río de los magueyes pequeños".

En la actualidad Aramecina pertenece a la Mancomunidad de Municipios Fronterizos (MAFRON), que busca gestionar y fortalecer alianzas para el desarrollo de estos pueblos olvidados.

\subsection{Datos demográficos y dinámica poblacional del municipio de Aramecina \\ El municipio de Aramecina es eminentemente rural, ninguna de sus comunidades reúne las características contempladas por el INE para considerarse urbana ${ }^{1}$, (Ver cuadro No.1).}

Cuadro No. 1. Aramecina: población por sexo, 2010

\begin{tabular}{|c|c|c|c|}
\hline Área & Hombres & Mujeres & Total \\
\hline Rural & 3,051 & 3,421 & 6,472 \\
\hline
\end{tabular}

Fuente: Instituto Nacional de Estadística (INE), Datos proyectados 2010.

De total de la población al 2010, el 47.1\%, eran hombres y $52.8 \%$, mujeres, con 5.4 habitantes promedio por vivienda. La emigración de los hombres hacia Estados Unidos u otros centros urbanos del país en busca de oportunidades, puede influenciar en el porcentaje inferior de hombres respecto a las mujeres (INE 2010).

Datos proyectados por el INE al 2010 muestran un estancamiento en el crecimiento poblacional con un total de 6,472 habitantes, en un periodo de 9 años sólo se presenta un incremento de 12 personas. Entre 1970 y el 2001 la población del municipio creció a un ritmo de $1.03 \%$ anual. Este crecimiento ocurrió principalmente en la cabecera municipal $2.11 \%$ y fue menor en el resto del municipio, $1.01 \%$ (ver cuadro No. 2).

1. El INE define el área urbana como aquellos lugares donde la población es mayor a 2,000 habitantes, hay servicio de agua por tubería, comunicación terrestre o servicio regular marítimo, escuela primaria completa de 6 grados, correo o telégrafo y que tengan al menos alcantarillado, alumbrado eléctrico o centro de salud. 
Cuadro No. 2. Aramecina: crecimiento poblacional entre 1971-2001

\begin{tabular}{|l|c|c|c|c|}
\hline \multicolumn{1}{|c|}{ Área } & 1971 & $\mathbf{2 0 0 1}$ & $\begin{array}{c}\text { Crecimiento } \\
\text { obser- } \\
\text { vado en el } \\
\text { período }\end{array}$ & $\begin{array}{c}\text { Tasa promedio } \\
\text { de crecimiento } \\
\text { anual observada }\end{array}$ \\
\hline Cabecera & 785 & 1,092 & 307 & $1.11 \%$ \\
\hline Aldeas & 3,909 & 5,287 & 1,378 & $1.01 \%$ \\
\hline Total & $\mathbf{4 , 6 9 4}$ & $\mathbf{6 , 3 7 9}$ & $\mathbf{1 , 6 8 5}$ & $1.03 \%$ \\
\hline
\end{tabular}

Fuente: Instituto Nacional de Estadística (INE), Censos de Población y Vivienda 1970 y 2001.

La mayoría de la población del municipio de Aramecina puede considerarse joven: el $86 \%$ es menor de 50 años, de los cuales el $46 \%$ son niños menores de 15 años, lo que indica una creciente demanda sobre los recursos del municipio. Las personas mayores de 50 años representan únicamente el 14\% de la población (INE, Censo 2001).

De acuerdo a los pobladores, esto está ocurriendo porque una gran cantidad de personas están migrando en busca de mejores oportunidades. Los hombres son los que generalmente migran, lo que explica porqué la población de mujeres es superior a la de los hombres lo que implica una composición familiar en la que la mujer es la jefe de familia.

La migración provoca una reducción de la mano de obra y a la vez cambios en las actividades económicas, aumentando aquellas que requieren menos mano de obra como la ganadería y reduciendo las que requieren más, como la producción agrícola.

\subsection{Cobertura escolar}

En Aramecina se ofrecen los siguientes niveles de enseñanza: pre-básico, básico, medio y diversificado. En el nivel pre básico están los jardines de niños oficiales, los jardines PROHECO y los Centros Comunitarios de Educación Prebásica (CCEPREB), en el nivel básico están las escuelas oficiales y las escuelas PROHECO. En el municipio operan un total de 43 centros educativos (ver cuadro No.3).

Para el año 2010 en los diferentes niveles educativos del municipio de Aramecina se atendió un total de 1,546 alumnos, el mayor número de alumnos y alumnas
Cuadro No. 3. Aramecina: centros educativos públicos y privados

\begin{tabular}{|l|c|}
\hline \multicolumn{1}{|c|}{ Centro Educativo } & No. en Funcionamiento \\
\hline Institutos Oficiales & 1 \\
\hline Escuelas Primarias Oficiales & 19 \\
\hline Escuelas PROHECO & 4 \\
\hline Jardines Oficiales & 6 \\
\hline Jardines PROHECO & 1 \\
\hline CCEPREB & 12 \\
\hline Centros Educativos privados & 0 \\
\hline Total & 43 \\
\hline
\end{tabular}

Fuente: Elaboración propia con datos de la Dirección Departamental de Educación de Valle.

se concentra en el nivel básico, siendo el nivel medio el que concentra el menor número.

Según datos de la Dirección Distrital de Educación No. 4 de Aramecina, en promedio, el 69\% de toda la población del municipio tiene algún nivel de educación primaria, pero sólo el $9 \%$ logra ascender al siguiente nivel de secundaria. En la cabecera municipal este porcentaje disminuye en el nivel de primaria (62\%) pero el $23 \%$ ha continuado la secundaria. En el resto del municipio la situación es inversa, 71\% de la población tiene algún nivel de primaria, pero apenas el 5\% continúa al nivel secundario (Dirección Distrital de Educación No. 4, 2009). Estas diferencias del bajo nivel educativo de la población en la cabecera y el resto del municipio deben tomarse en cuenta para el diseño de cualquier estrategia de desarrollo municipal.

\subsection{Migración y remesas en el municipio de Aramecina}

La migración en Honduras es un fenómeno producido por la falta de oportunidades en donde los ciudadanos puedan desarrollar sus capacidades y ejercer a cabalidad sus derechos, por lo que se ven obligados a buscarlas fuera de las fronteras nacionales.

En el tema de migración de los y las hondureñas hacia Estados Unidos y España, es necesario tener presente las salidas de forma irregular, ayudadas por coyotes, como también el drama que enfrentan los familiares que se quedan con las deudas, pagando altos intereses a los prestamistas, siendo víctimas de los usureros o estafado- 
res, firmando letras de cambio en blanco, hipotecando y hasta entregando bienes inmuebles dando margen a todo tipo de extorsiones; todo esto a cambio de las promesas para ser trasladados a los países de destino.

Aramecina es un municipio pequeño en el cual existen escasas oportunidades de trabajo por lo que sus habitantes han sido influenciados con las tendencias de los salvadoreños a migrar a los Estados Unidos en forma ilegal por medio de individuos que se dedican al tráfico de personas, a quienes entregan grandes cantidades de dinero a cambio del sueño americano, lo que implica en muchas ocasiones el riesgo de su propia vida.

Según el Censo de Población y Vivienda de 2001, el municipio de Aramecina se encuentra entre los $10 \mathrm{mu}$ nicipios con mayor proporción de personas viviendo en otro país. Ello implica una mayor probabilidad de recibir remesas, lo cual permite mejorar el ingreso y por ende las condiciones de vida de su población" (IDH, 2006: 31).

Investigaciones realizadas por FUNDER en 2003 indican que Aramecina presenta un crecimiento en la construcción y mejoramiento de calidad de viviendas, que refleja la influencia de las remesas. Asimismo esto estimula la migración entre los jóvenes de la comunidad y concuerda con la reducción de la población en el municipio (Fundación Vida, 2004: 17).

En un estudio realizado por PNUD, FLACSO encontró que en Aramecina un 3.60\% de los hogares entrevistados se reporta, por lo menos un miembro con intención de migrar; de estos, el 50\% lo logró y el 50\% falló en el intento. El 100\% de quienes no lograron emigrar fue por causa de deportación (Informe Municipal ODM: 42).

El 41\% de los encuestados en Aramecina, reportó recibir remesas. El ó la jefa de hogar son los principales receptores de las remesas (115) lo que representa el $76.20 \%$. El cuadro No. 4 muestra los porcentajes de tendencia de quienes reciben las remesas (Informe $\mathrm{Mu}$ nicipal ODM: 42).
Cuadro No. 4. Municipio de Aramecina: personas que recibe las remesas

\begin{tabular}{|l|c|}
\multicolumn{1}{|c|}{ Miembro del hogar } & Porcentaje \\
\hline Jefe(a) de hogar & 76.2 \\
\hline Esposo(a) & 17.2 \\
\hline Hijos & 4.7 \\
\hline Otro parientes & 2.7 \\
\hline
\end{tabular}

Fuente: Encuestas de Hogares PNUD-FLACSO, Agosto 2007.

\section{Resultados de investigación}

\subsection{Contexto general del Instituto Ángel G. Her- nández}

El Instituto "Ángel G. Hernández" fue fundado en el año de 1978, inicia bajo una modalidad semioficial, donde los padres de familia pagaron parte de los costos del centro educativo, es hasta el año de 1986 mediante acuerdo ejecutivo No. 4343 EP 86 del 3 de septiembre de 1986 que se oficializó.

El instituto se encuentra ubicado a 2 kilómetros de la frontera con El Salvador, inicio sus labores en un local prestado por la municipalidad funcionando con cuatro secciones de ciclo común, dos secciones de primer curso, una de segundo y una de tercer curso, las cuales se atendían en jornada matutina. A partir del año 2006 el instituto comenzó a funcionar en un local propio, en las afueras del casco urbano en el caserío El Llano del Pueblo, aldea La Peña.

El Instituto "Ángel G. Hernández" atiende en la actualidad las siguientes ramas de estudio: Ciclo Común de Cultura General y Bachillerato en Ciencias y Letras, su población estudiantil oscila entre 240 y 300 alumnos, distribuidos en 10 secciones, 3 de primer curso, 3 de segundo curso y 2 de tercer curso de Ciclo Común de Cultura General además una sección de I de Bachillerato en Ciencias y Letras y una sección de II de Bachillerato en Ciencias y Letras; de las diez secciones se atienden 5 en jornada matutina y 5 en jornada vespertina (ver cuadro No.5). 
Cuadro No. 5. Instituto "Ángel G. Hernández": distribución de alumnos por curso año 2010

\begin{tabular}{|l|c|c|c|}
\multicolumn{1}{c|}{ Curso } & Señoritas & Varones & Total \\
\hline I CCCG & 55 & 31 & 86 \\
\hline II CCCG & 34 & 30 & 64 \\
\hline III CCCG & 35 & 30 & 65 \\
\hline I Bach CC Y LL & 11 & 8 & 19 \\
\hline II Bach CCY LL & 15 & 4 & 19 \\
\hline Total & 150 & 103 & 253 \\
\hline
\end{tabular}

Fuente: Elaboración propia en base a datos de la Secretaría del instituto.

5.2 Efecto psicosocial de la migración en las familias de los y las alumnas del instituto Ángel G. Hernández y su incidencia en el rendimiento educativo

Para abordar la temática del impacto psicosocial, causada por la migración, es necesario referirnos al fenómeno migratorio como el desplazamiento geográfico de un lugar a otro de personas, generalmente por causas económicas o sociales.

El fenómeno de la migración no es nocivo, sin embargo, si no se la realiza de manera planificada, ordenada y por la vía legal, puede causar daños irreparables al tejido social del país, entre ellos la desintegración familiar, que conlleva a serios problemas psicológicos a los niños, niñas y adolescentes traduciéndose en bajo rendimiento escolar, alcoholismo, drogadicción, pandillerismo, intentos de suicidio, entre otras patologías sociales.

En el desarrollo de este estudio se aplicó una encuesta a los estudiantes de educación media del Instituto "Ángel G. Hernández" del municipio de Aramecina, departamento de Valle, en el sur de Honduras, cuyo objetivo es determinar los efectos psicosociales de la migración que afectan el rendimiento educativo, se entrevistaron a 239 jóvenes y se encontraron los siguientes resultados:

\subsubsection{Sexo de los y las estudiantes encuestadas}

Se encuestaron 239 estudiantes, de los cuales el $61 \%$ son mujeres y el $39 \%$ son hombres, superando en porcentaje la población femenina (ver gráfico No.1); en parte el porcentaje responde a que los hombres no se matriculan porque desde temprana edad se incorporan como mano de obra familiar o se ven obligados a viajar para buscar trabajo y así poder sacar adelante a su familia.

\subsubsection{Progenitor migrante}

De los 239 alumnos entrevistados más del 50\% de ellos tienen un progenitor o ambos en condición de migrante, la mayoría ha emigrado a los Estados Unidos en busca de fuentes de trabajo que generen ingresos para satisfacer las necesidades básicas de su familia.

De manera general la mayor frecuencia de migración se presenta entre los jefes de hogar en donde el padre es la persona que migra en mayor porcentaje (60\%), en tanto que la madre representa sólo el 20\% de los casos entrevistados (ver gráfico No.2).

Este fenómeno social conlleva por un lado la pérdida de capital humano y social dentro de la comunidad, también afecta de manera profunda la estructura familiar, especialmente al incrementarse la feminización de las jefaturas de hogar y causar el despoblamiento de las comunidades, pero a su vez la migración contribuye al bienestar de la economía familiar impactando favorablemente en la economía nacional a través de las remesas.

\section{Gráfico No. 1. Instituto Ángel G. Hernández: población estudiantil encuestada por sexo, en porcentajes}

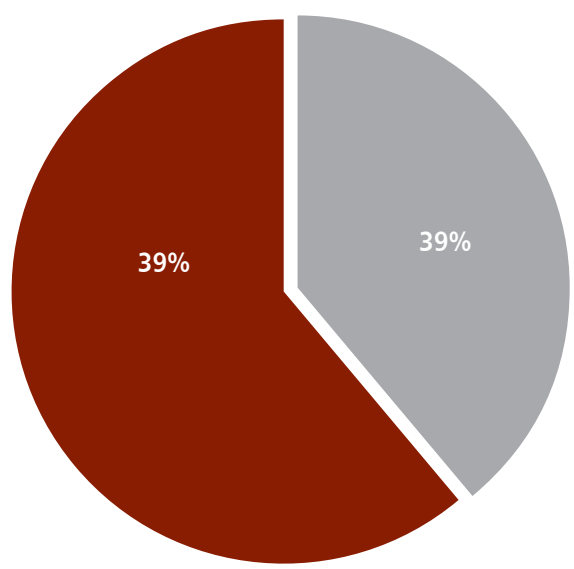

Fuente: Elaboración propia en base a los resultados de encuesta aplicada en Abril del 2010. 
Gráfico No. 2. Instituto Ángel G. Hernández: Progenitor Migrante por Estudiante, en porcentaje.

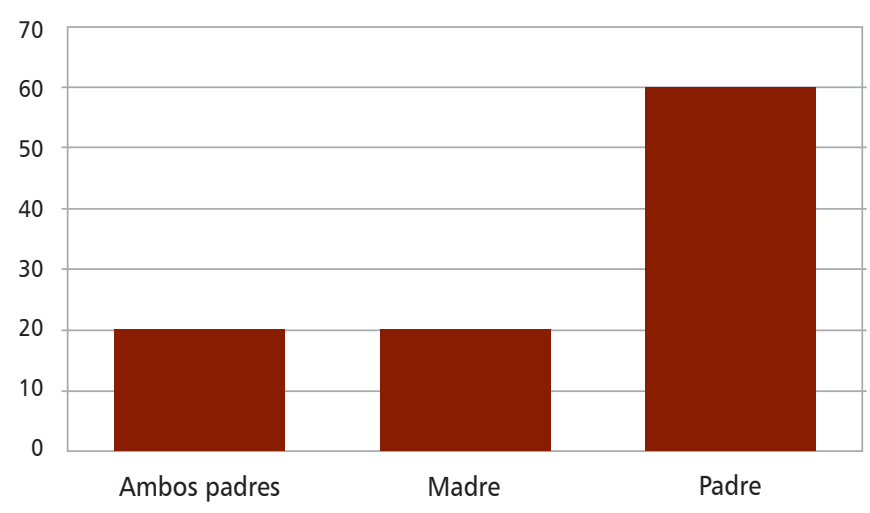

Fuente: Elaboración propia en base a los resultados de encuesta aplicada en abril del 2010

Se puede afirmar que la migración si bien genera algunos beneficios inmediatos termina a la larga por debilitar la estructura familiar, los niños, niñas y jóvenes muchas veces no entienden porque sus padres se han ido y empiezan a desarrollar sentimientos que van desde el odio hasta la indiferencia hacia ellos. El dinero enviado por los padres logra satisfacer sus necesidades económicas pero no su compañía y afecto, ni sustituye el amor. La mayoría de esos niños, niñas y jóvenes están en desamparo sin alguien que los guíe o aconseje en esta etapa tan difícil de la adolescencia.

\subsubsection{Familiares de los y las alumnas en condición de} migrantes

Según datos obtenidos en la encuesta se puede decir que el $75 \%$ de la población estudiantil tiene al menos un familiar migrante, $30 \%$ corresponde al padre de familia, $10 \%$ a la madre, $10 \%$ al padre y madre a la vez $12 \%$ a los hermanos y el resto a familiares como abuelos, tíos y primos (ver cuadro No.6).

Esta situación pone en alerta al personal docente y administrativo del instituto porque podría ser una causa de los problemas de conducta y bajo rendimiento académico de los y las alumnas.
Cuadro No. 6. Instituto Ángel G. Hernández: familiar del alumno del en condición de migrante

\begin{tabular}{|l|c|c|}
\hline $\begin{array}{l}\text { Familiar (es) en condición de } \\
\text { migrante }\end{array}$ & Entrevistados & $\%$ \\
\hline Padre & 72 & 30.1 \\
\hline Madre & 24 & 10.0 \\
\hline Padre + Madre & 24 & 10.0 \\
\hline Abuela & 3 & 1.2 \\
\hline Abuelo (a) & 2 & 0.8 \\
\hline Hermano(a) & 31 & 12.9 \\
\hline Tío (a) & 21 & 8.7 \\
\hline Primo & 2 & 0.8 \\
\hline Ninguno & 60 & 25.1 \\
\hline Total & 239 & 100.0 \\
\hline
\end{tabular}

Fuente: Encueta aplicada a las y los alumnos del Instituto Ángel G. Hernández año 2010.

En el Instituto “Ángel G. Hernández” por medio de los departamentos de Consejería y Orientación se trata de orientar a los estudiantes con problemas académicos y de conducta, buscando las posibles causas y soluciones para mejorar el proceso de enseñanza aprendizaje. El cuadro No.7 presenta los resultados obtenidos al revisar los registros académicos y de conducta de los entrevistados con familiar migrante.

En estos resultados se puede apreciar que en cierta manera sí influye la presencia de los padres en el rendimiento académico de los y las alumnas, ya que los padres están pendientes de las tareas y apoyan a sus hijos e hijas en las diferentes responsabilidades asignadas para su aprendizaje.

Cuadro No. 7. Instituto Ángel G. Hernández: rendimiento académico de los y las alumnas con padres migrantes

\begin{tabular}{|c|c|c|c|c|c|}
\hline \multirow{2}{*}{$\begin{array}{l}\text { Familiar } \\
\text { ausente }\end{array}$} & \multicolumn{4}{|c|}{ Asignaturas Reprobadas } & \multirow[b]{2}{*}{ Repitente } \\
\hline & Ninguna & 1 & 2 & 3 ó más & \\
\hline Ambos padres & 9 & 3 & 5 & 6 & 1 \\
\hline Padre & 41 & 15 & 5 & 11 & 0 \\
\hline Madre & 13 & 2 & 4 & 3 & 1 \\
\hline Total & 63 & 20 & 14 & 20 & 2 \\
\hline
\end{tabular}

Fuente: Elaboración propia con registros del Departamento de Consejería y Orientación del IAGH, 2010. 
Los datos no reflejan una diferencia tan marcada en relación al rendimiento académico de los alumnos/as con padres presentes y aquellos que tienen padres migrantes esto quizá esté relacionado con el tiempo de ausencia de los padres, ya que se ha comprobado que entre más tiempo tiene el padre ausente en el hogar, el alumno y alumna tiende a recuperar su nivel académico, permitiendo relaciones más estrechas, además hoy en día la tecnología juega un papel muy importante ya que la comunicación con los migrantes es más fácil.

Sin embargo, la migración de los padres incide de una u otra manera en el rendimiento académico de los hijos sobre todo por la preocupación que llegan a tener los menores por sus padres.

\subsubsection{Tiempo de ausencia del progenitor}

El tiempo de ausencia del progenitor con mayor frecuencia es entre 9 y 12 años con un 34\%, seguido de un periodo entre 12 ó más años con 20\%. Es importante destacar que más del $71.66 \%$ de los alumnos y alumnas entrevistadas, sus padres migrantes tienen más de cinco años de ausencia en el hogar, factor que ha influido al hecho que estos jóvenes se hayan adaptado a su ausencia (ver cuadro No. 8).

Cuadro No. 8. Instituto Ángel G. Hernández: tiempo de ausencia del progenitor en el hogar de los y las alumnas

\begin{tabular}{|l|c|c|}
\hline \multicolumn{1}{|c|}{ Tiempo de ausencia } & Entrevistados & $\%$ \\
\hline No sabe & 2 & 1.6 \\
\hline Menos de 1 año & 9 & 7.5 \\
\hline Entre 1 y 4 años & 23 & 19.1 \\
\hline Entre 5 y 8 años & 21 & 17.5 \\
\hline Entre 9 y 12 años & 41 & 34.1 \\
\hline Entre 12 ó más años & 24 & 20.0 \\
\hline Total & 120 & 100.0 \\
\hline
\end{tabular}

Fuente: Entrevistas individuales a los alumnos del Instituto Ángel G. Hernández año 2010.

Como ha corroborado la UNESCO, el rendimiento escolar es un fenómeno en el que influyen múltiples factores causales. Luego de haber revisado la literatura y estudios realizados en distintos tipos de establecimientos educativos (escuelas, colegios, universidades) a nivel mundial, se ha definido un listado de variables relevantes para determinar el rendimiento de los estudiantes de educación media, dado que migró uno de sus padres; entre estos factores se identifica: el interés de la familia, nivel de ingreso familiar, nivel de educación de los padres, repetición de años, acceso a texto y materiales, habilidades innatas, tiempo utilizado para aprender, entre otras; las mismas que servirán para demostrar cuan significativo puede ser para un estudiante de educación media, el hecho que su(s) padre(s) tengan que migrar, en busca de una mayor estabilidad económica.

La migración va de la mano con la pobreza, donde el costo elevado de la vida y la escasez de fuentes de trabajo hacen que los jefes del hogar opten por emprender el viaje a lugares donde el ofrecimiento de empleos y los mejores salarios les den la oportunidad de llevar lo básico a sus hogares, quedando los y las niñas y jóvenes sin la seguridad que da la familia, obstaculizando el desarrollo emocional de estos.

\subsubsection{Problemas de conducta presentados por los y las} alumnas del Instituto Ángel G. Hernández

Dentro de los problemas presentados por los y las alumnas con padres migrantes, basándose en los controles llevados por el Departamento de Orientación del instituto se puede afirmar que sólo un 15\% de ellos ha sido tratado en este departamento y de estos el $33 \%$ ha sido registrado y tratado por condiciones de salud y el $44 \%$ por su rendimiento académico, y un $22.2 \%$ por problemas de conducta (Departamento de Orientación del Instituto Ángel G. Hernández, año 2010).

Al revisar el libro de actas de la Consejería del instituto refleja un resultado sorprendente en donde el 61\% de los alumnos y alumnas registrados en las actas vienen de hogares con padres migrantes (ver gráfico No.3), dentro de los problemas presentados los más comunes son: los pleitos con los compañeros siendo un indicativo de agresión tanto verbal como física, también la desobediencia a la autoridad del centro educativo, no entrar a clases o no presentar las tareas asignadas, actitud malcriada y problemas de uniforme incompleto. 
Gráfico No. 3. Instituto Ángel G. Hernández: porcentaje de alumnos que presentan problemas de disciplina, 2010

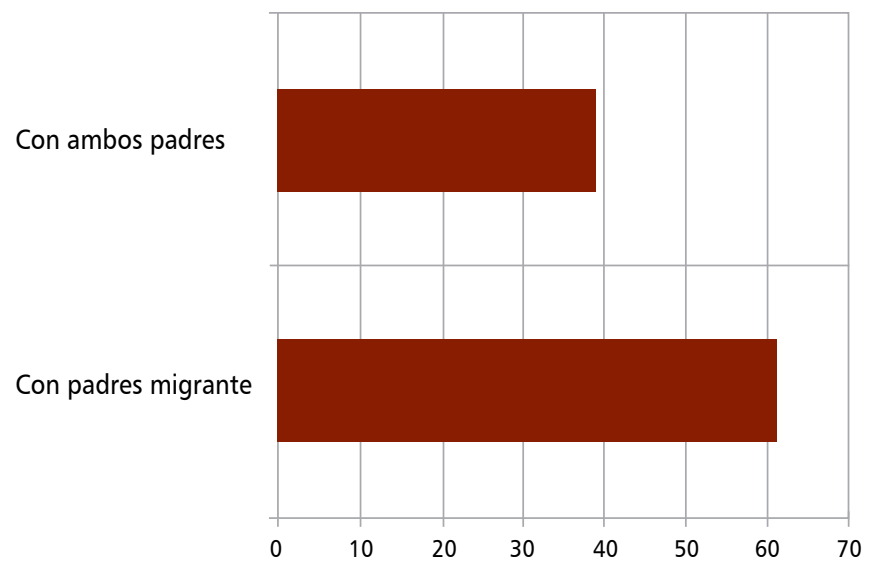

Fuente: Elaboración propia en base a Libro de actas de Consejería del Instituto Ángel G. Hernández año 2010.

Se puede apreciar que el $64 \%$ de los y las alumnas con problemas de ausencia del progenitor está entre 1-8 años, disminuyendo el porcentaje en los alumnos cuyos padres llevan ausente entre 9 y 12 años (ver cuadro No. 9).

Hay jóvenes que pueden asimilar y aceptar la ausencia de sus padres con menos dificultad; no obstante, hay otros que por determinadas características de personalidad, circunstancias y dinámica familiar la interpretan como abandono y desprotección, lo que puede llegar a repercutir en su autoestima al sentir que no son merecedores de atención y afecto, y como consecuencia se les dificulta integrarse socialmente, razón por la cual poseen menos recursos para enfrentar la vida.

En el instituto se presentó el caso de una alumna quien después de que su padre migró y las abandonó, se

Cuadro No. 9. Instituto Ángel G. Hernández: tiempo de ausencia de los padres de los alumnos con problemas disciplinarios 2010.

\begin{tabular}{|l|c|}
\hline Tiempo de ausencia de los padres & $\begin{array}{c}\text { Alumnos con problemas } \\
\text { disciplinarios }\end{array}$ \\
\hline Entre 1 y 4 años & 40.9 \\
\hline Entre 5 y 8 años & 22.7 \\
\hline Entre 9 y 11 años & 9.1 \\
\hline Entre 12 ó más años & 27.3 \\
\hline Total & 100.0 \\
\hline
\end{tabular}

Fuente: Entrevistas individuales a los alumnos del Instituto Ángel G. Hernández año 2010. castigó a sí misma y dejo de hablar, ni siquiera se comunicaba con su madre y todos los cinco años que curso sus estudios tuvo la misma actitud, fue hasta en II de bachillerato que comenzó a hablar con sus compañeros y profesores.

Es importante señalar que el $73 \%$ de los alumnos que presentaron problemas de disciplina en el año 2010 vive con su mamá siendo su padre el familiar migrante, no hubo ningún caso de algún estudiante con los dos padres migrantes lo que viene a reforzar la hipótesis que los/as alumnas con ambos padres emigrantes tienden a madurar en forma precoz.

\subsubsection{Impacto en los alumnos y alumnas por ausencia} de un familiar

$\mathrm{Al}$ preguntarles a los y las estudiantes como ha impactado en sus vidas la ausencia del familiar en condición de emigrante obtuvimos los siguientes resultados (ver cuadro No. 10).

- Impactos positivos de la migración entre los y las estudiantes del Instituto Ángel G. Hernández del municipio de Aramecina

Según versión de los alumnos y alumnas encuestadas desde el punto de vista económico han mejorado sus condiciones materiales de vida, les ha permitido estudiar y continuar sus estudios a nivel superior, han mejorado la infraestructura del hogar, tienen lo básico para vivir. Con respecto a los jóvenes que dicen que han sido impactados positivamente por la ausencia de un familiar en condición de

Cuadro No. 10. Instituto Ángel G. Hernández: tipo de impacto en los alumnos y alumnas con padres migrantes, 2010.

\begin{tabular}{|l|c|c|}
\hline \multicolumn{1}{|c|}{ Tipo de Impacto } & Entrevistados & $\%$ \\
\hline Positivo & 22 & 18.3 \\
\hline Negativo & 59 & 49.2 \\
\hline No hay cambios & 24 & 20.0 \\
\hline no sabe & 15 & 12.5 \\
\hline Total & 120 & 100 \\
\hline
\end{tabular}

Fuente: Elaboración propia con las entrevistas individuales a los alumnos del Instituto Ángel G. Hernández año 2010. 
migrante, que es el $22 \%$, expresaron que sienten emociones como la alegría y la felicidad cuando reciben las remesas.

Al irse mi mamá como migrante estamos viviendo muy bien como Dios nos tiene".

Rony Pastor Maldonado Romero

Como nuestros padres se establecieron en Estados Unidos hoy tenemos papeles para poder viajar legalmente a continuar nuestros estudios.

Andy Patricia y Rosy Mary Rivera Banegas

Nosotras nacimos en Estados Unidos, ya que nuestros padres migraron para ese país y somos ciudadanas americanos.

Jennifer Villatoro Hernández y Jessica Anastasia Cruz

- Impactos negativos de la migración entre los y las estudiantes del Instituto Ángel G. Hernández del municipio de Aramecina.

Los impactos negativos de la migración son más que todo a nivel emocional (sentimientos de tristeza, depresión, rebeldía) o conductual: intromisión en maras o pandillas juveniles, embarazo adolescente, repitencia escolar, entre otros.

En el caso de las personas que han sido impactadas negativamente por causa de la ausencia de un familiar en condición de emigrante, el 59\%, expresó sentir emociones como tristeza, abandono, falta de cariño y frustración por el hecho de extrañar a su pariente.

"Me siento pésimo, bien mal porque lo extraño, son 15 años de no estar a mi lado... y es demasiado".

Jessica Sarahí Rivera Rivera

"No tengo su apoyo se fueron y se olvidaron de mi, si no fuera por mi abuela...."

Ruth Bery Banegas

De una u otra manera los jóvenes son impactados emocionalmente y esto puede afectarles más sobre todo porque se encuentran en edad adolescente.

\section{Conclusiones}

- En general una alta cantidad de jóvenes consideran que la causa principal de la ausencia de su padre se debe al abandono de la comunidad, la falta de fuentes de trabajo y la pobreza, situaciones que motivan la migración en la zona con el fin de lograr un trabajo con el cual obtener dinero para poder ayudar y sostener económicamente a su familia.

- La mayor parte de los familiares en condición de migrante de los y las alumnas del Instituto Ángel G. Hernández lleva una ausencia entre 5 y 12 años por lo que la mayoría de los jóvenes con padres migrantes ya se ha acostumbrado a la ausencia de su familiar.

- Los problemas disciplinarios más frecuentes presentados por los y las alumnas con padres migrantes están referidos a pleitos y riñas hasta la rebeldía con lo que confirmamos que la migración si tienen repercusiones psicosociales en los jóvenes.

- El 49\% de los/as jóvenes entrevistados considera que la migración de sus padres ha provocado un impacto negativo en sus vidas al carecer de cariño, consejos y cuidados que no pueden ser sustituidos por el dinero recibido.

\section{Bibliografía}

- INE, 2001 XVI Censo de Población y V de Vivienda.

- INE, 2010 Proyecciones de Población.

- Instituto Ángel G. Hernández (2010) Libro de actas del departamento de Consejería

- Instituto Ángel G. Hernández (2010) Cuadros No. 1 de la Secretaría.

- Instituto Ángel G. Hernández (2010) Hojas de incidencias llevadas por el Departamento de Orientación del Instituto.

- María Antonia Parra y otros (2009) Explosión Migratoria y sus Efectos en el Rendimiento Académico, editorial y lugar de edición.

- Observatorio de las Metas del Milenio (2009) Informe Municipal de Aramecina, ODM.

- PNUD-IDH: 2006, Más allá de la escazes; poder, pobreza y crisis mundial del agua.

- Fundación Vida 2004. Diagnóstico Ambiental Municipal Participativo y Plan de Accion con la facilitacion de la Fundación Vida-Prrac Dl. 